\title{
Analysis of optomechanical coupling in two-dimensional square lattice phoxonic crystal slab cavities
}

\author{
Said El-Jallal, ${ }^{1,4}$ Mourad Oudich, ${ }^{1}$ Yan Pennec, ${ }^{1}$ Bahram Djafari-Rouhani, ${ }^{1}$ Vincent Laude, ${ }^{2}$ Jean-Charles Beugnot, ${ }^{2}$ Alejandro \\ Martínez, ${ }^{3}$ José María Escalante ${ }^{3}$ and Abdelkader Makhoute ${ }^{4}$ \\ ${ }^{1}$ Institut d'Electronique, Microélectronique et Nanotechnologie, UMR CNRS 8520, Université Lille 1, Villeneuve d'Ascq, France \\ ${ }^{2}$ Institut FEMTO-ST, UMR CNRS 6174, Université de Franche-Comté and CNRS, Besançon, France \\ ${ }^{3}$ Nanophotonics Technology Center, Universitat Politècnica de València, València, Spain \\ ${ }^{4}$ Physique du Rayonnement et de l'Interaction Laser Matière, Université de Moulay Ismail, Meknès, Morocco
}

(Received 13 July 2013; published 7 November 2013)

\begin{abstract}
We theoretically investigate phonon-photon interaction in cavities created in a phoxonic crystal slab constituted by a two-dimensional (2D) square array of holes in a silicon membrane. The structure without defects provides 2D band gaps for both electromagnetic and elastic waves. We consider two types of cavities, namely, an L3 cavity (a row of three holes is removed) and a cross-shape cavity, which both possess highly confined phononic and photonic localized modes suitable for enhancing their interaction. In our theoretical study, we take into account two mechanisms that contribute to optomechanical interaction, namely, the photoelastic and the interface motion effects. We show that, depending on the considered pair of photonic and phononic modes, the two mechanisms can have similar or very different magnitudes, and their contributions can be either in or out of phase. We find out that only acoustic modes with a specific symmetry are allowed to couple with photonic cavity modes. The coupling strength is quantified by two different methods. In the first method, we compute a direct estimation of coupling rates by overlap integrals, while in the second one, we analyze the temporal modulation of the resonant photonic frequency by the phonon-induced acoustic vibrational motion during one acoustic period. Interestingly, we obtain high optomechanical interaction, with the coupling rate reaching more than $2.4 \mathrm{MHz}$ for some specific phonon-photon pairs.
\end{abstract}

DOI: 10.1103/PhysRevB.88.205410

PACS number(s): 43.40.+s, 42.70.Qs, 42.79.Jq

\section{INTRODUCTION}

Periodic structures called photonic ${ }^{1,2}$ and phononic $^{3-5}$ crystals are known to be highly dispersive media for the propagation of electromagnetic and acoustic waves, respectively. These structures can induce frequency stop bands, which make them very efficient tools for the sake of many potential applications in wave guiding, filtering, confinement, and sensing. Combining photonic and phononic crystals into a single structure provides a new toolbox to control and manipulate light and sound simultaneously, the so-called phoxonic crystal (PxC). The latter has been receiving increasing interest for the last few years, as it can exhibit simultaneous photonic and phononic band gaps. ${ }^{6-12}$ Maldovan and Thomas ${ }^{6}$ demonstrated this property for an infinite two-dimensional (2D) crystal made of a square or hexagonal lattice of holes in silicon. Sadat-Saleh et al. ${ }^{7}$ extended this investigation to lithium niobate $\left(\mathrm{LiNbO}_{3}\right)$ and considered more general lattices. These papers demonstrated the existence of an absolute phononic band gap together with a photonic band gap associated with either the transverse electric (TE) or the transverse magnetic (TM) polarization of light. Bria et al. ${ }^{8}$ demonstrated the possibility of obtaining an absolute photonic gap as well, by using the anisotropy of sapphire in the microwave regime. Later, the possibility of obtaining dual phononic-photonic band gaps was investigated in crystal slabs of silicon perforated with holes. ${ }^{9,10}$ It was demonstrated that simultaneous photonic and phononic band gaps can occur for square and hexagonal lattices, but not for the triangular lattice. Additionally, absolute photonic band gaps can be found for more complicated lattices such as boron nitride. ${ }^{10}$ Shortly after, it was theoretically demonstrated that a periodic array of silicon pillars deposited on thin homogeneous silica $\left(\mathrm{SiO}_{2}\right)$ membrane can also be a promising phoxonic system for the three most common lattices, i.e., square, triangular, and honeycomb, ${ }^{11}$ especially as concerns the flexibility in the choice of the geometrical parameters.

Recently, optomechanical (OM) interaction has been investigated in different structures based on slabs using well constructed cavities. Safavi-Naeini et al. ${ }^{13,14}$ observed a strong OM coupling between co-localized resonant phonons and photons with a high quality factor inside a well-tailored tapered linear cavity defect in a silicon slab. Using membrane acoustic wave excitation, Fuhrman et al. ${ }^{15}$ were able to demonstrate experimentally the acoustic modulation of the resonant optical frequency of a confined mode inside a cavity. Gavartin et al. ${ }^{16}$ reported, both theoretically and experimentally, strong $\mathrm{OM}$ coupling inside a 2D optomechanical slab cavity. Alongside these works, other groups reported strong OM interaction between phonon and photons in nanobeam one-dimensional (1D) structures, both theoretically and experimentally. ${ }^{17-19}$ However, most of these works considered localized phonons and photons that are not necessarily trapped inside a photonic and/or a phononic band gap. Using the dual phonon-photon band gap property, one can expect to introduce a defect inside the phoxonic crystal that can confine both phonons and photons for the purpose of enhancing their OM interaction. ${ }^{12,20}$ Recently, we have demonstrated that high phonon-photon coupling can be achieved by localizing modal frequencies inside the band gaps of a 1D multilayer structure, ${ }^{21}$ and of a 2D infinite PxC..$^{22,23}$

In the present paper, we investigate the $\mathrm{OM}$ interaction inside a phoxonic crystal slab constituted by a $2 \mathrm{D}$ square lattice array of holes drilled in a silicon membrane. By appropriately choosing the dimensions of the structure, it can display photonic and phononic band gaps for guided modes in the optical telecom range and at gigahertz acoustic 
frequencies. In recent papers, it was proven that, for specific geometrical parameters, this structure possesses an absolute phononic band gap together with a photonic band gap of a given symmetry. 9,10 This results in an opportunity to confine elastic waves and linearly polarized electromagnetic fields inside linear or point defects, in view of enhancing their interaction. We study the case of two simple cavities, namely, an L3 cavity obtained by removing three holes along the [100] direction in the crystal and a cross-shaped cavity obtained by removing four holes adjacent to a central removed hole. The coupling between phonons and photons results from two mechanisms that contribute to the total OM interaction: the photoelastic (PE) effect and the deformation of interfaces caused by acoustic strain. ${ }^{19,22}$ The strength of the OM coupling is estimated in two different but complementary ways. The first method is the so-called coupling rate calculation, based on the overlap integral of acoustic and optical wave fields. ${ }^{19}$ The second method is based on the calculation of the acoustic modulation of the photonic cavity mode, where the resonant frequency of the photonic mode is calculated at several instants during one period of the acoustic vibration. ${ }^{21-23} \mathrm{~A}$ major point in this investigation is to stress that only modes of a given symmetry are suitable for providing nonvanishing optomechanical interaction. All calculations performed in this article, i.e., photonic and phononic dispersion curves, and OM coupling rate estimations, were performed using the finite element method (COMSOL ${ }^{\circledR}$ Multiphysics).

The outline of the paper is as follows. We first discuss in Sec. II the photonic and phononic dispersion curves in order to define partial and full band gaps and then consider the photonic modes of the L3 cavity and of the cross-shaped cavity. In Sec. III, we describe in detail the two methods of calculation used for the estimation of the OM interaction strength, namely, the coupling rate calculations and the acoustic modulation of the resonant photonic frequency during one acoustic period. For each cavity, the coupling rate is calculated between photonic modes and all phononic modes in a wide range of frequencies, and the most efficient combinations are identified and characterized. The modulation of photonic modes is then presented for these most efficient combinations, for each cavity. Finally, conclusions are drawn in Sec. IV.

\section{PXC SLAB AND PHOTONIC CAVITY MODES}

We investigate a phoxonic crystal slab composed of a square lattice array of cylindrical air holes drilled in a silicon membrane. For a lattice constant $a=540 \mathrm{~nm}$, this structure has been demonstrated to be a phoxonic crystal operating in the gigahertz regime and the telecom range for phonons and photons, respectively. ${ }^{9-11}$ Systematic calculations showed that specific geometrical parameters can be chosen to provide dual phonon/photon band gaps in this structure. Quantitatively, for a filling fraction $f=0.58$ (hole radius $r=0.43 a$ ) and a slab thickness $h=0.6 a$, band structure calculations show the stop bands for both photons and phonons summarized in Fig. 1. Figure 1(a) displays the phononic band structure in which we have separated modes according to their symmetry with respect to the middle plane of the plate. The even and odd symmetries are respectively distinguished with the red and the black colors. The band structure indicates an absolute phononic band gap, highlighted in gray and extending from 5.86 to $6.19 \mathrm{GHz}$. There is also a wider partial gap for even modes in the frequency range $5-6.76 \mathrm{GHz}$ (red area). Distinguishing modes with respect to their symmetry is important when searching for phononic modes that can couple with photonic modes and will be discussed in the following section. Figure 1(b) displays the photonic band structure in which even modes appear in red and odd modes appear in blue. Two band gaps are furthermore observed for even photonic modes, in the wavelength ranges $1435.4-1565.2 \mathrm{~nm}$ and $963.1-1068 \mathrm{~nm}$ (red areas), while only one gap is obtained for odd modes, in the wavelength range 1167.1-1331.7 nm (blue area).

We then study the effect of the insertion of two different defect cavities. The first one, known as an L3 cavity, is created
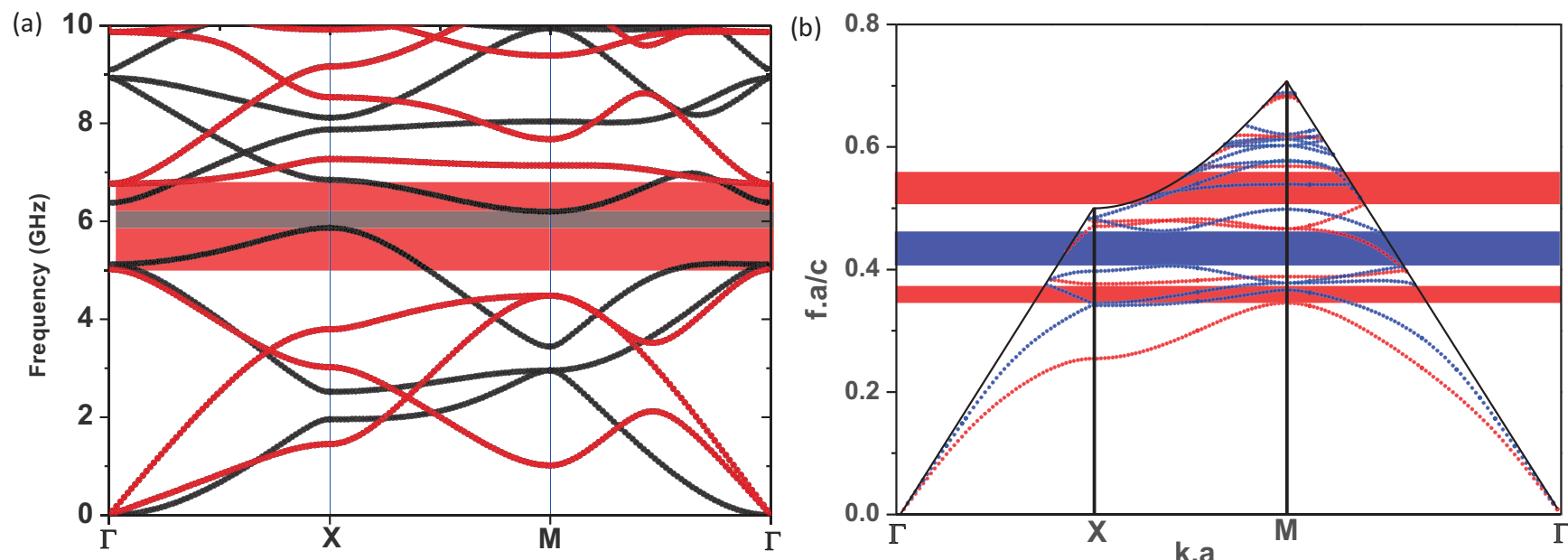

FIG. 1. (Color online) Phononic and photonic dispersion relation computed using the finite element method. (a) Phononic band structure for a crystal slab of silicon perforated with a square lattice array of air holes. The radius of the holes and the thickness of the slab are, respectively, $r=0.43 a$ and $h=0.6 a$, where $a=540 \mathrm{~nm}$ is the lattice constant. Bands for modes with even symmetry (respectively, odd symmetry) are drawn in red (resp., black), while the absolute gap is shown in gray. (b) Photonic band structure with even and odd symmetry bands plotted in red and blue colors, respectively. 


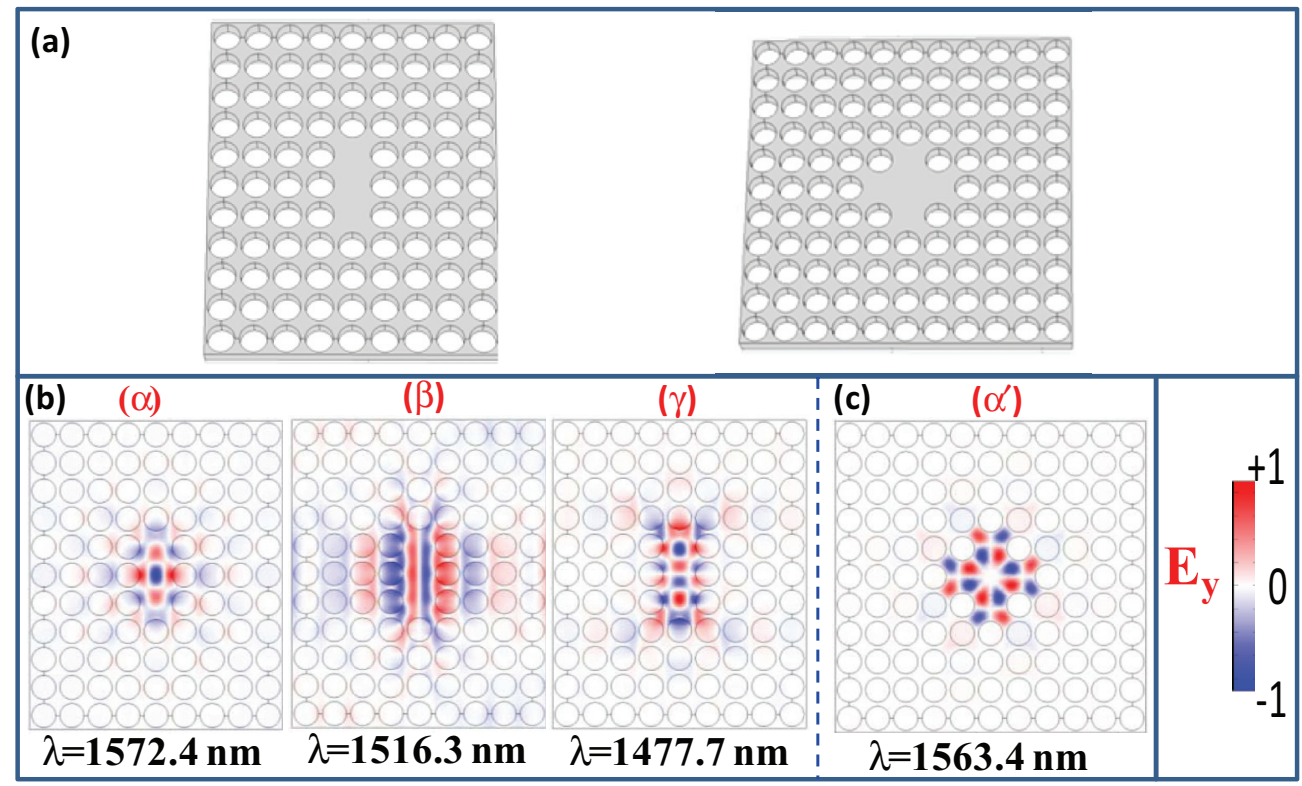

FIG. 2. (Color online) (a) Schematic representation of the L3 and the cross cavities created inside the square lattice phoxonic crystal slab. Distribution of the electric field $E_{y}$ of (b) the three photonic modes $\alpha, \beta$, and $\gamma$ inside the L3 cavity and (c) the photonic mode $\alpha^{\prime}$ inside the cross cavity.

by removing three holes on the same row, while the second cavity is obtained by creating a cross in the middle of the super cell, as depicted in Fig. 2. The reason behind the choice of the L3 cavity is that it can provide us with several photonic and phononic modes inside band gaps. In fact, we first investigated a simple L1 cavity (only one hole removed) and found that it provides only one photonic mode that can couple with only one phononic mode at the limit of the partial gap, far away from the absolute phononic gap. Additionally, the optical transmission spectrum through the L1 cavity showed a broad peak covering almost the whole photonic band gap, which means that this mode is not well confined. By choosing the L3 cavity, then, we expect to find more photonic modes with higher confinement, giving stronger coupling with phononic modes. In parallel, we propose a cross-shaped cavity, which provides photonic modes with higher quality factors than the L3 cavity.

To study the phononic and photonic cavity modes inside the two cavities, we perform dispersion calculations using the "super-cell" technique. In the super cell, the cavity is surrounded by four holes, meaning that, due to the periodic boundary conditions, each cavity is separated from its neighbor by eight holes. This precaution insures isolation of the modes of neighboring cavities. For the L3 cavity, we obtain three localized photonic modes, denoted $\alpha, \beta$, and $\gamma$, located inside the first partial band gap for even symmetry modes, at $1477.7 \mathrm{~nm}, 1516.3 \mathrm{~nm}$, and $1572.4 \mathrm{~nm}$, respectively. Their respective electric field components $E_{y}$ are presented in Fig. 2(b), showing a strong localization inside and in the vicinity of the cavity. Regarding the cross cavity, we find one localized cavity mode inside the partial photonic band gap denoted $\alpha^{\prime}$ at $1563.4 \mathrm{~nm}$, for which the electric field $E_{y}$ is depicted in Fig. 2(c).

In parallel, to estimate the quality factors of the photonic modes, we perform transmission calculations. Indeed, the photonic modes of the cavities give rise to peaks in the transmission coefficient, and their quality factor can be estimated from the width of the corresponding peak. In this way, we obtain quality factors of less than 1000 for the photonic modes in the L3 cavity and above 3200 for the mode in the cross-shaped cavity.

\section{STRENGTH OF THE OM COUPLING}

\section{A. OM coupling rates}

As considered in previous studies, ${ }^{19,22,23}$ two main mechanisms contribute to the OM coupling, producing a local variation of the dielectric permittivity: the moving interfaces (MI) and the PE effects. ${ }^{24}$ The latter effect results from the variation of the dielectric permittivity due to the acoustic strain

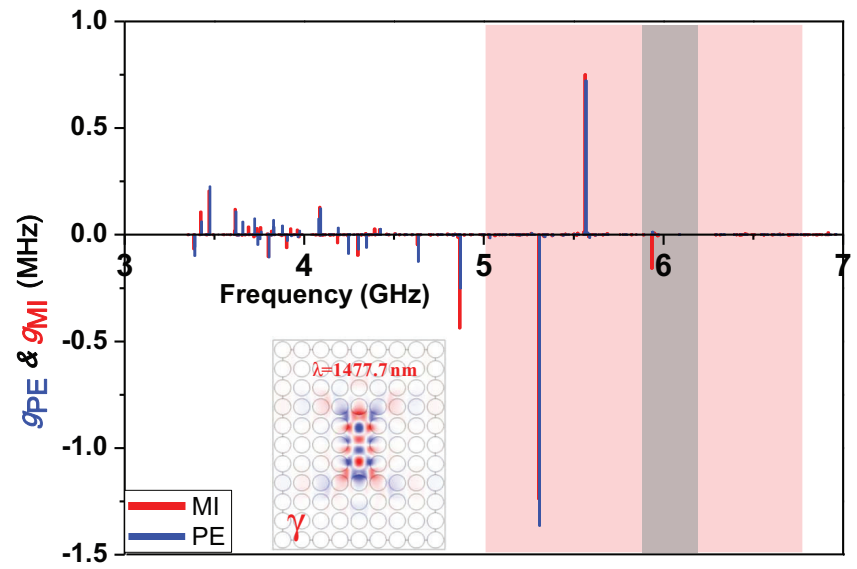

FIG. 3. (Color online) Calculation of the OM coupling rate between photonic mode $\gamma$ and all phononic modes in the range 3-7 GHz. Red (blue) thin bars correspond to the MI (PE) contribution to the coupling rate. 


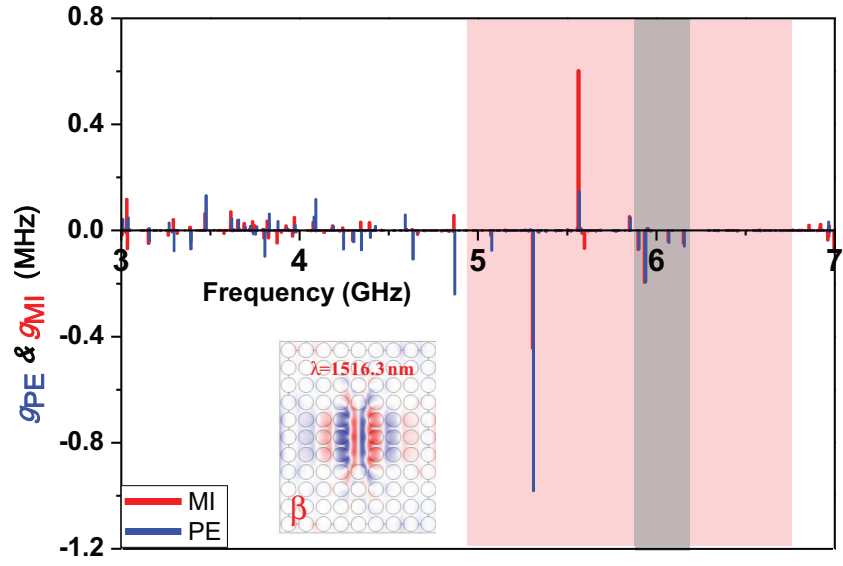

FIG. 4. (Color online) Calculation of the OM coupling rate between photonic mode $\beta$ and all phononic modes in the range 3-7 GHz. Red (blue) thin bars correspond to the MI (PE) contribution to the coupling rate.

inside the bulk of the material, while the MI mechanism comes from the boundaries' motions, resulting in a variation of the dielectric permittivity in the vicinity of the interfaces between two different media - silicon and air in our case. In our study, we used two different methods to estimate the strength of the phonon-photon coupling. The first one is based on the calculation of the OM coupling rates, and the second is based on the modulation of the photonic mode by the acoustic strain during one acoustic period.

In the first method, the coupling rate describes the frequency shift induced by the zero-point motion of the mechanical field of the phonon. The formulas that allow for the calculation of the OM coupling are given by Chan et al. ${ }^{19}$ for both PE and MI effects:

$$
\begin{gathered}
g_{\mathrm{PE}}=-\frac{\omega}{2} \frac{\langle E|\delta \varepsilon| E\rangle}{\int_{V} \mathbf{E} \cdot \mathbf{D} d V} \sqrt{\hbar / 2 M_{\mathrm{eff}} \Omega .} \\
g_{\mathrm{MI}}=-\frac{\omega}{2} \frac{\oint_{\partial V}(\mathbf{U} \cdot \mathbf{n})\left(\Delta \varepsilon \mathbf{E}_{\|}^{2}-\Delta \varepsilon^{-1} \mathbf{D}_{\perp}^{2}\right) d S}{\int_{V} \mathbf{E} \cdot \mathbf{D} d V} \sqrt{\hbar / 2 M_{\mathrm{eff}} \Omega .}
\end{gathered}
$$

Here, $\sqrt{\hbar / 2 M_{\mathrm{eff}} \Omega}$ is the zero-point motion of the oscillator, where the effective mass is given by $M_{\text {eff }}=\int \rho\left(\frac{\|\mathbf{U}\|}{\|\mathbf{U}\|_{\max }}\right)^{2} d V$, and $\mathbf{U}$ is the normalized displacement field (with $\max \|\mathbf{U}\|=1), \mathbf{n}$ is the outward normal to the boundary, $\mathbf{E}$ is the electric field, $\mathbf{D}$ is the electric displacement field, and $\varepsilon$ is the dielectric permittivity. In these relations, $\left(\Delta \varepsilon=\varepsilon_{\text {silicon }}-\varepsilon_{\text {air }}\right)$ and $\left(\Delta \varepsilon^{-1}=\varepsilon_{\text {silicon }}^{-1}-\varepsilon_{\text {air }}^{-1}\right)$, and $\delta \varepsilon_{\mathrm{ij}}=-\varepsilon_{0} n^{4} p_{\mathrm{ijkl}} S_{\mathrm{kl}}$, where $p_{\mathrm{ijkl}}$ are the photoelastic tensor components, $n$ is the refractive index of silicon, and $S_{k l}$ represent the strain tensor

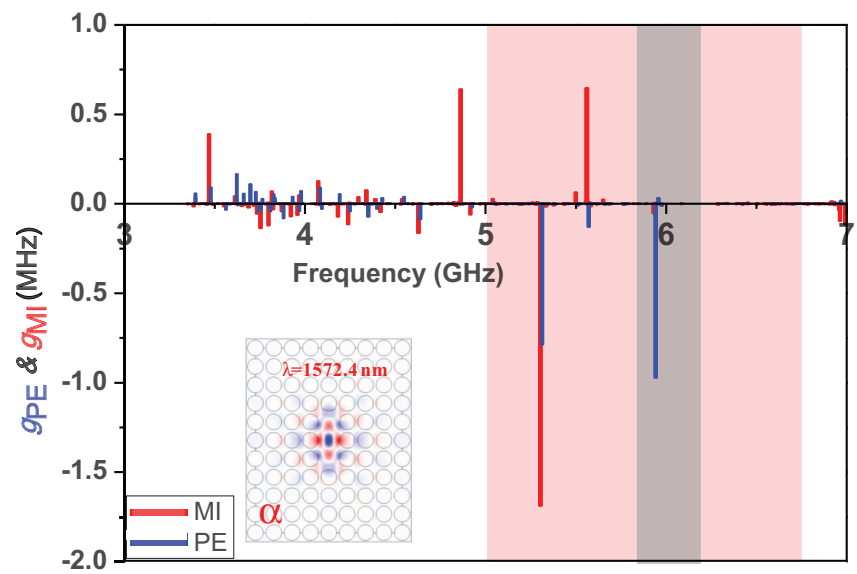

FIG. 5. (Color online) Calculation of the OM coupling rate between photonic mode $\alpha$ and all phononic modes in the range 3-7 GHz. Red (blue) thin bars correspond to the MI (PE) contribution to the coupling rate.

components. Thus, we obtain

$$
\begin{aligned}
\langle E|\delta \varepsilon| E\rangle= & -\varepsilon_{0} n^{4} \int\left[2 \operatorname{Re}\left\{E_{x}^{*} E_{y}\right\} p_{44} S_{x y}\right. \\
& +2 \operatorname{Re}\left\{E_{x}^{*} E_{z}\right\} p_{44} S_{x z}+2 \operatorname{Re}\left\{E_{y}^{*} E_{y}\right\} p_{44} S_{y z} \\
& +\left|E_{x}\right|^{2}\left(p_{11} S_{x x}+p_{12}\left(S_{y y}+S_{z z}\right)\right) \\
& +\left|E_{y}\right|^{2}\left(p_{11} S_{y y}+p_{12}\left(S_{x x}+S_{z z}\right)\right) \\
& \left.+\left|E_{z}\right|^{2}\left(p_{11} S_{z z}+p_{12}\left(S_{x x}+S_{y y}\right)\right)\right] d V .
\end{aligned}
$$

A symmetry analysis based on these expressions shows that only phononic modes with even symmetry with respect to the middle plane of the slab can couple to the optical modes. For instance, with a phononic mode of odd symmetry, the above integral takes equal values with opposite signs on each half of the cavity, such that the integral over the whole structure vanishes. This is true at least in the result of the first-order perturbation theory presented above, where OM coupling is based on a one-phonon process. Two- or many-phonon processes are still possible for other symmetries of the modes, though their strength should remain small for physically acceptable acoustic strain fields. Similar conclusions hold for other optomechanical structures reported in the literature, although such symmetry considerations have not been emphasized before.

In the second method, we estimate the OM coupling from the modulation of the photonic resonant frequency during one acoustic period for a given strain level. Numerically, we begin with the calculation of the monochromatic steady-state

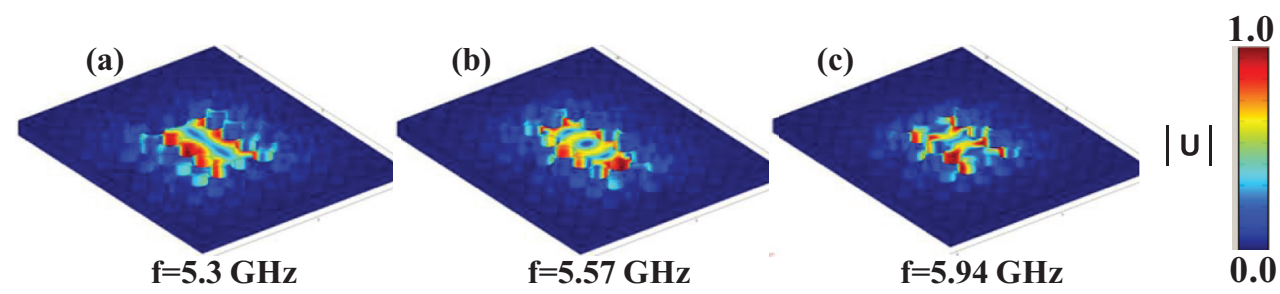

FIG. 6. (Color online) Modulus of the displacement field for the three localized phononic modes (a), (b), and (c) inside the L3 cavity. 
TABLE I. Total OM coupling rate in L3 cavity for both PE and MI effects, for the photonic mode and three phononic modes.

\begin{tabular}{lcccc}
\hline \hline & \multicolumn{4}{c}{ Photonic mode } \\
\cline { 2 - 5 }$g=g_{\mathrm{PE}}+g_{\mathrm{MI}}(\mathrm{MHz})$ & & $\alpha$ & $\beta$ & $\gamma$ \\
\hline Phononic mode & $a$ & 2.47 & 1.43 & 2.6 \\
& $b$ & 0.52 & 0.75 & 1.47 \\
& $c$ & 1.02 & 0.39 & 0.14 \\
\hline \hline
\end{tabular}

photonic mode by applying a given strain field. The acoustic period is sampled into several time steps, and the photonic cavity modes are calculated in the perturbed structure at each time step. This procedure enables us to obtain the evolution of the electromagnetic frequency during one acoustic period. For the sake of computational facilities, the modulations of the photonic frequencies are calculated by assuming that the maximum displacement amplitude in the cavity is fixed at $1 \%$ of the lattice period. Actually, this deformation is much greater than more realistic values of $10^{-4}$ to $10^{-6}$, even if the acoustic excitation is generated by an acoustic source. For this reason, the method provides only the relative rates of the OM couplings when comparing the results from one photon-phonon pair to another or when comparing the contributions of the PE and MI mechanisms. Also, we checked that the trends obtained with this method are coherent with the coupling rates derived from Eqs. (1) and (2). However, the absolute values of the coupling rates obtained in both methods are not comparable, since the maximum amplitudes of the displacement field in the cavity are taken differently.

We start with the L3 cavity and the calculation of the OM coupling rate for each photonic mode, $\alpha, \beta$, and $\gamma$, interacting with all the phononic cavity modes located between 3 and $7 \mathrm{GHz}$. Within this wide acoustic frequency range, many phononic modes are present in the cavity, some of which are resonant (below $5 \mathrm{GHz}$ ) and others of which belong to the partial $(5-6.76 \mathrm{GHz})$ or to the absolute $(5.86-6.19 \mathrm{GHz})$

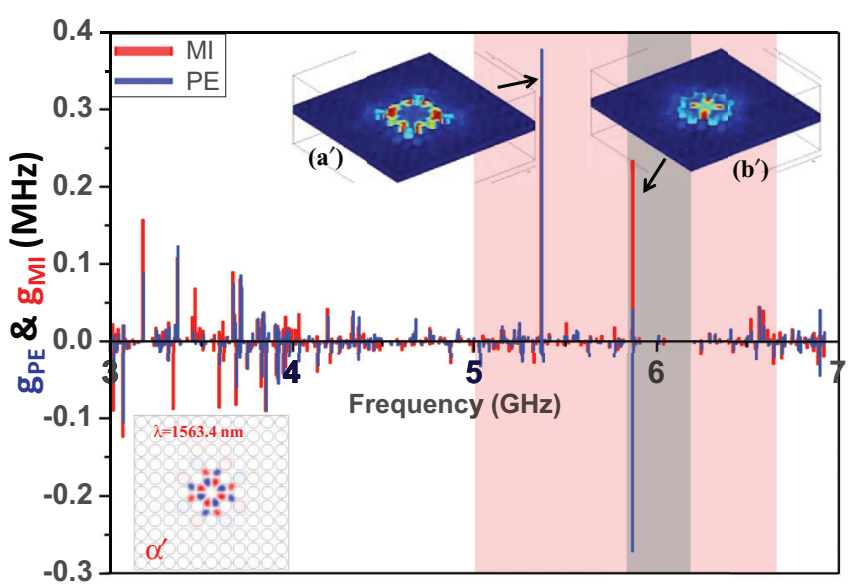

FIG. 7. (Color online) Calculation of the OM coupling rate between photonic mode $\alpha^{\prime}$ and all phononic modes in the range 3-7 GHz for the cross-shaped cavity. Red (resp., blue) thin bars correspond to the MI (resp., PE) contribution to the coupling rate. (Inset): Total displacement field for modes $a^{\prime}$ and $b^{\prime}$ inside the cross cavity
TABLE II. OM coupling rate for both PE and MI effects, together with the total OM effect for the two phononic modes inside the band gap for the cross cavity.

\begin{tabular}{lrcc}
\hline \hline$g=g_{\mathrm{PE}}+g_{\mathrm{MI}}(\mathrm{MHz})$ & \multicolumn{3}{c}{ Photonic mode $\alpha^{\prime}$} \\
\hline Phononic modes & $g_{\mathrm{PE}}$ & $g_{\mathrm{MI}}$ & $g=g_{\mathrm{PE}}+g_{\mathrm{MI}}$ \\
$a^{\prime}$ & 0.38 & 0.32 & 0.7 \\
$b^{\prime}$ & -0.27 & 0.23 & -0.04 \\
\hline \hline
\end{tabular}

phononic band gaps. Coupling rate calculations are presented in Figs. 3, 4, and 5 for both the MI and the PE effects, corresponding to the interaction between all phononic modes and the previous three photonic modes. In each of these three figures, we plotted the contributions of the MI and of the PE effects with red and blue bars, respectively. From these figures, one can see that three phononic modes with even symmetry inside the partial (red area) or the absolute (gray area) phononic band gap present a significant OM coupling rate. These three phononic modes are located at $5.3 \mathrm{GHz}, 5.57 \mathrm{GHz}$, and $5.94 \mathrm{GHz}$, and their displacement field distributions, reported in Fig. 6, show they are well confined inside the cavity. Additionally, the good confinement of the phononic modes in the cavities is confirmed by the high quality factor (above $10^{6}$ ) obtained in the calculation of the phononic transmission through the structure. For the sake of clarity, we have summarized the values of the coupling rate between the three photonic modes and the three phononic modes in Table I. Compared to equivalent optomechanical slab structures, our coupling rates are slightly higher than the values found in the literature. In Ref. 16, Gavartin et al. experimentally obtained a maximum of the coupling rate of $0.53 \mathrm{MHz}$ for a cavity inside a silicon plate drilled with holes arranged according to a hexagonal lattice. Safavi-Naeini et $a l .{ }^{13}$ gave a theoretical value of the coupling rate equal either to $1.8 \mathrm{MHz}$ for a "snowflake" crystal slab structure ${ }^{14}$ or to $2 \mathrm{MHz}$ in a slotted two-dimensional photonic crystal cavity. The highest value was reported by Chan et al..$^{19}$ for a 1D nanobeam structure, which had a theoretical value of $5.4 \mathrm{MHz}$ and an experimental value of $1.1 \mathrm{MHz}$.

From Figs. 3, 4, and 5 and Table I, one can draw the following conclusions:

(i) The third phononic mode, denoted (c), which is located inside the absolute band gap, presents a low OM coupling rate with photonic modes $\beta$ and $\gamma$. However, this phonon (c) shows a strong coupling with the photonic mode $\alpha$, mainly thanks to the PE effect, while the MI effect remains weak.

(ii) The phononic mode (b), located inside the even phononic band gap, presents the highest value for the full OM coupling rate $\left(g=g_{\mathrm{PE}}+g_{\mathrm{MI}}=1.47 \mathrm{MHz}\right)$ with the photonic mode $\gamma$ (Table I). This is due to an equivalent contribution of both PE and MI effects.

(iii) The resonant phononic mode (a) gives the highest full OM coupling overall, with values higher than $2.4 \mathrm{MHz}$ for photonic modes $\alpha$ and $\gamma$, and larger than $1.4 \mathrm{MHz}$ for photonic mode $\beta$. The coupling in this case is mainly strengthened by the additive contributions of the PE and the MI effects, since coupling rates $\mathrm{g}_{\mathrm{MI}}$ and $\mathrm{g}_{\mathrm{PE}}$ have the same sign and significant values. One can remark that with the two pairs of phonon/photon $(a, \gamma)$ and $(a, \beta)$, the PE effect contribution 


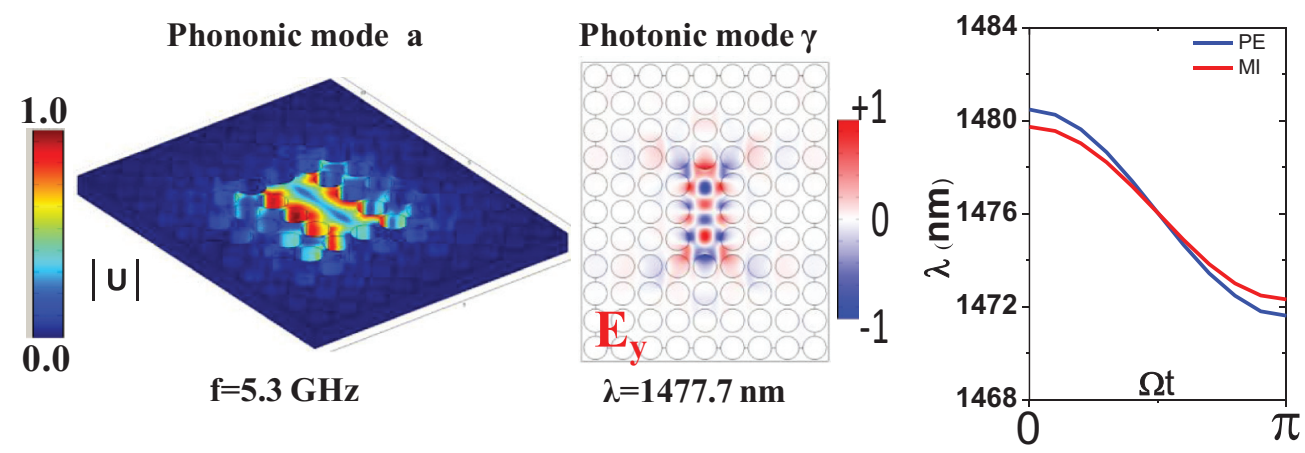

FIG. 8. (Color online) OM modulation of the photonic cavity wavelength for mode $\gamma$ at $1477.7 \mathrm{~nm}$ along half of the acoustic period of the phononic mode a at 5.3 GHz. The MI and the PE effects are represented with red and blue solid lines, respectively.

is higher than the MI effect contribution, while the opposite situation is observed with pair $(a, \alpha)$.

As in the case of the L3 cavity, we have investigated the OM coupling for the cross cavity. The coupling evaluation is done between photonic mode $\alpha^{\prime}$ with all phononic modes in the frequency range 3-7 GHz. The results of the calculations of the coupling rates $\mathrm{g}_{\mathrm{PE}}$ and $\mathrm{g}_{\mathrm{MI}}$ are presented in Fig. 7 . We find two phononic modes, denoted $a^{\prime}$ inside the partial band gap $(5.35 \mathrm{GHz})$ and $b^{\prime}$ inside the absolute band gap $(5.87 \mathrm{GHz}$ ) (see the displacement field distributions in the inset of Fig. 7). Both phononic modes give high values for each separate contribution by PE and MI. Nevertheless, due to the signs of the two effects, the total effect can be either additive, as for mode $a^{\prime}$, leading to a high OM coupling, or subtractive, as for mode $b^{\prime}$, resulting in a low OM coupling. For the cross cavity, the values of the coupling rate between the photonic mode and the two phononic modes are summarized in Table II.

\section{B. OM modulation of the resonant photonic frequency}

As described in Sec. III A, another estimation of the phonon/photon interaction strength can be achieved by calculating the modulation of the resonant photonic frequency by the acoustic strain during one acoustic period. For this calculation, we will consider the maximum of the elastic displacement inside the cavity to be $1 \%$. We present in the following the results for the L3 and the cross cavity, which correspond to the strongest phonon-photon couplings, and compare them with the previous coupling rate data.
For the L3 cavity, the highest OM coupling rate is obtained for the phonon/photon pair $(a, \gamma)$. Figure 8 presents the photonic frequency modulation of mode $\gamma$ due to the phonon strain (a) during one half of the acoustic period. One can see from Fig. 8 that $46 \%$ of the full OM interaction is due to the MI effect, while $54 \%$ comes from the PE contribution, in good agreement with coupling rate calculations. Quantitatively, the magnitude of photonic mode wavelength shift is about $8.87 \mathrm{~nm}$ for the PE effect and $7.43 \mathrm{~nm}$ for the MI mechanism.

For the cross cavity, the highest OM coupling rate was obtained considering the interaction between phononic mode $a^{\prime}$ and photonic mode $\alpha^{\prime}$. Figure 9 represents the modulation of the photonic mode, where $55 \%$ of the OM interaction is due to the PE effect, and 45\% comes from the MI effect. Quantitatively, the magnitude of photonic wavelength modulation for a maximum of $1 \%$ elastic displacement inside the cavity is about $3.5 \mathrm{~nm}$ for the PE effect and $2.84 \mathrm{~nm}$ for the MI effect. These two effects are, as previously, additive and in good agreement with the coupling rate calculations shown in the previous section.

\section{CONCLUSION}

In this paper, we have theoretically investigated the OM interaction in cavities created in a phoxonic crystal slab composed of a 2D square lattice of air holes presenting band gaps for both photonic and phononic modes. The creation of two kinds of defect cavities, i.e., an L3 and a cross cavity, leads to several localized photonic and phononic modes, which propose several possibilities to confine simultaneously
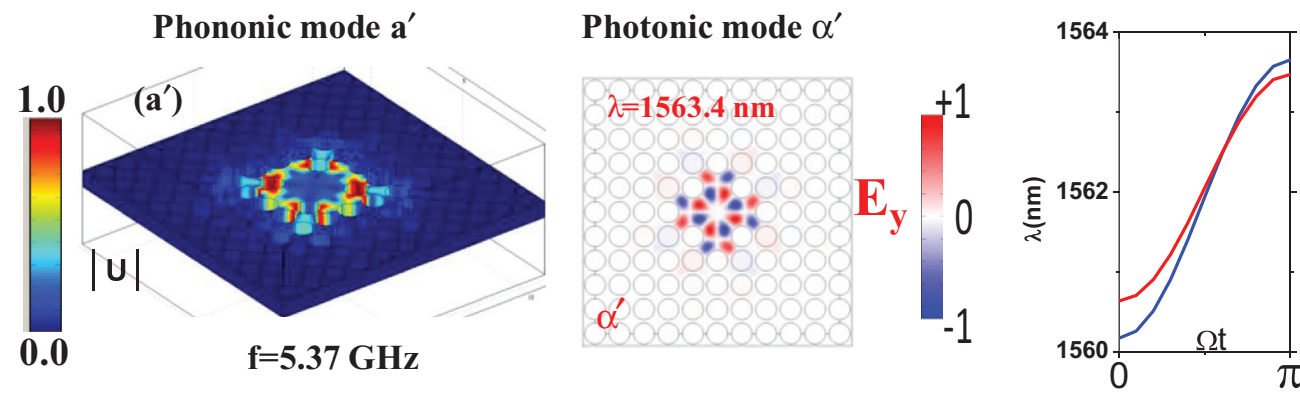

FIG. 9. (Color online) OM modulation of the photonic cavity wavelength for mode $\alpha^{\prime}$ at $1563.4 \mathrm{~nm}$ along half of the acoustic period of phononic mode $a^{\prime}$ at $5.37 \mathrm{GHz}$. The MI and the PE effects are represented with red and blue solid lines, respectively. 
phonons and photons and to enhance their interaction. In particular, phononic modes can be either in the absolute phononic band gap or in the partial gap of even symmetry with respect to the middle plane of the slab. Indeed, we have shown that the coupling with the optical mode is efficient as soon as the symmetry of the phononic mode is even. The OM coupling has been calculated considering two main mechanisms: the MI and the PE effects. Two methods of calculation have been considered, the calculation of the coupling rate, on the one hand, and the acoustic modulation of the resonant photonic frequency during one acoustic period, on the other hand. For both cavities, we show that at least one phonon/photon pair leads to a high optomechanical effect. The origin of the high OM coupling has been discussed in each case, considering separately the contributions of the MI and the PE effects. The most efficient couplings are obtained when the MI and the PE effects are additive and equivalent in magnitude. We find coupling rates up to $2.47 \mathrm{GHz}$, which are slightly larger than values found in the literature for similar slab structures.
Additionally, our phoxonic structure presents a phononic cavity mode inside the absolute band gap that leads to a high acoustic Q factor. Finally, we show that the strength of the OM couplings is higher in the L3 cavity than in the cross cavity. This conclusion has to be moderated by the fact that the cross cavity presents an optical quality factor of about 3200 , i.e., about four times higher than that of the L3 cavity. We are currently improving these quality factors by adjusting the positions of the holes around the cavity. Our results show that defect cavities in 2D PxC slabs displaying a simultaneous band gap for photons and phonons can be a suitable platform for building advanced optomechanical components supporting enhanced photon-phonon interactions.

\section{ACKNOWLEDGMENTS}

The authors acknowledge the support of the European Commission Seventh Framework Programs (FP7) under the FET-Open project TAILPHOX N 233883.
${ }^{1}$ E. Yablonovitch, J. Opt. Soc. Am. B 10, 283 (1993).

${ }^{2}$ T. F. Krauss, R. M. De la Rue, and S. Brandt, Nature 383, 699 (1996).

${ }^{3}$ M. M. Sigalas and E. N. Economou, J. Sound Vib. 158, 377 (1992).

${ }^{4}$ M. S. Kushwaha, P. Halevi, L. Dobrzynski, and B. Djafari-Rouhani, Phys. Rev. Lett. 71, 2022 (1993).

${ }^{5}$ Y. Pennec, J. O. Vasseur, B. Djafari-Rouhani, L. Dobrzynski, and P. A. Deymier, Surf. Sci. Rep. 65, 229 (2010).

${ }^{6}$ M. Maldovan and E. L. Thomas, Appl. Phys. B 83, 595 (2006).

${ }^{7}$ D. Bria, M. B. Assouar, M. Oudich, Y. Pennec, J. Vasseur, and B. Djafari-Rouhani, J. Appl. Phys. 109, 014507 (2011).

${ }^{8}$ S. Sadat-Saleh, S. Benchabane, F. I. Baida, M. P. Bernal, and V. Laude, J. Appl. Phys. 106, 074912 (2009).

${ }^{9}$ S. Mohammadi, A. A. Eftekhar, A. Khelif, and A. Adibi, Opt. Express 18, 9164 (2010).

${ }^{10}$ Y. Pennec, B. Djafari-Rouhani, E. H. El Boudouti, C. Li, Y. El Hassouani, J. O. Vasseur, N. Papanikolaou, S. Benchabane, V. Laude, and A. Martinez, Opt. Express 18, 14301 (2010).

${ }^{11}$ Y. El Hassouani, C. Li, Y. Pennec, E. H. El Boudouti, H. Larabi, A. Akjouj, O. Bou Matar, V. Laude, N. Papanikolaou, A. Martinez, and B. Djafari Rouhani, Phys. Rev. B 82, 155405 (2010).

${ }^{12}$ V. Laude, J. C. Beugnot, S. Benchabane, Y. Pennec, B. DjafariRouhani, N. Papanikolaou, J. M. Escalante, and A. Martinez, Opt. Express 19, 9690 (2011).

${ }^{13}$ A. H. Safavi-Naeini and O. Painter, Opt. Express 18, 14926 (2010).
${ }^{14}$ A. H. Safavi-Naeini, T. P. Mayer Alegre, M. Winger, and O. Painter, Appl. Phys. Lett. 97, 181106 (2010).

${ }^{15}$ D. A. Fuhrmann, S. M. Thon, H. Kim, D. Bouwmeester, P. M. Petroff, A. Wixforth, and H. J. Krenner, Nature Photon. 5, 605 (2011).

${ }^{16}$ E. Gavartin, R. Braive, I. Sagnes, O. Arcizet, A. Beveratos, T. J. Kippenberg, and I. Robert-Philip, Phys. Rev. Lett. 106, 203902 (2011).

${ }^{17}$ M. Eichenfield, J. Chan, R. M. Camacho, K. J. Vahala, and O. Painter, Nature 462, 78 (2009).

${ }^{18}$ M. Eichenfield, J. Chan, A. H. Safavi-Naeini, K. J. Vahala, and O. Painter, Opt. Express 17, 20078 (2009).

${ }^{19}$ J. Chan, A. H. Safavi-Naeini, J. T. Hill, S. Meenehan, and O. Painter, Appl. Phys. Lett. 101, 081115 (2012).

${ }^{20}$ M. Maldovan and E. L. Thomas, Appl. Phys. Lett. 88, 251907 (2006).

${ }^{21}$ I. E. Psarobas, N. Papanikolaou, N. Stefanou, B. Djafari-Rouhani, B. Bonello, and V. Laude, Phys. Rev. B 82, 174303 (2010).

${ }^{22}$ Q. Rolland, M. Oudich, S. El-Jallal, S. Dupont, Y. Pennec, J. Gazalet, J. C. Kastelik, G. Lévêque, and B. Djafari-Rouhani, Appl. Phys. Lett. 101, 061109 (2012).

${ }^{23}$ M. Oudich, S. El-Jallal, Y. Pennec, G. Leveque, B. Djafari-Rouhani, Q. Rolland, S. Dupont, J. Gazalet, J.-C. Kastelik, and A. Makhoute, in IEEE International Ultrasonics Symposium (IUS), Dresden, 7-10 October 2012 (IEEE, Piscataway, 2012), pp. 2466-2469.

${ }^{24} \mathrm{~A}$. Yariv and P. Yeh, Optical Waves in Crystals (Wiley, New York, 1984). 